\title{
Contribución al conocimiento del falso fraguado. Efectos de la langbeinita cálcica producida durante la clinkerización
}

\author{
TOMAS VAZQLEZ HORENo, Dr. en Ciencias Quimicas \\ OIGA FERNANDEZ PESA, Perito Industrial Químico
}

IETCC

\section{NOTA PRELIMINAR}

El presente trabajo ha sido publicado por el IETCC como CUADERNO DE INVESTIGACION, con el n.' 34 y fecha de julio de 1981. No obstante se ha considerado oportuno el incluirlo en esta Revista MATERIALES DE CONSTRUCCION. Los resultados que se obtienen de la investigación realizada, así como las determinaciones experimentales llevadas a cabo, son aún incompletos; es necesario complementar estas determinaciones para comprobar el efecto que ejercen sobre el falso fraguado y el "aterronamiento" de los cementos una serie de variables, tales como: temperatura, humedades relativas, superficie específica, álcalis, $\mathrm{CaO}$ libre, etc.

En el IETCC actualmente se está trabajando en esos aspectos con el fin de aclarar, en lo posible, la influencia que ejercen aquellos parámetros sobre el "aterronamiento" y el falso fraguado en diversos cementos portland.

\section{INTRODUCCION}

Son numerosas las hipótesis que se han expuesto para tratar de explicar el falso fraguado. Una gran parte de estas hipótesis se basa en la transformación del yeso hemihidrato (producido por deshidratación del yeso dihidrato durante la molienda) en yeso dihidrato durante los primeros minutos del amasado.

No obstante, en numerosas ocasiones se comprobó experimentalmente que cementos formados con hemihidrato, en vez de yeso, no acusaban el fenómeno del falso fraguado.

En el Instituto Eduado Torroja de la Construcción y del Cemento se venía observando que, cementos presentando el falso fraguado contenían singenita $\left(\mathrm{CaSO}_{4} \cdot \mathrm{K}_{2} \mathrm{SO}_{4} \cdot \mathrm{H}_{2} \mathrm{O}\right)$, aunque la presencia de este compuesto no siempre suponía que el cemento que lo contenía diera falso fraguado (1). Asimismo, se observó en ensayos llevados a cabo sobre cementos "recientes" y que daban fraguados normales, que al cabo de pocos días acusaban un falso fraguado muy marcado. Si posteriormente, y con un intervalo de pocos días esos mismos cementos se volvían a ensayar, ya no daban fraguado anormal.

(1) Tomás Vázquez Moreno y A. Ruiz de Gauna; Comunicación privada (Informe Técnico IETcc) 
Con el fin de aclarar el mecanismo que producía los efectos, anteriormente mencionados, en el IETCC se llevó a cabo un amplio estudio, partiendo de la hipótesis de que el azufre procedente del fuel (o del carbón), que se emplea en los hornos de cemento, se combina con los álcalis del crudo dando principalmente, $\mathrm{K}_{2} \mathrm{SO}_{4}$ (arcanita), $\mathrm{Na}_{2} \mathrm{SO}_{4} \cdot 3 \mathrm{~K}_{2} \mathrm{SO}_{4}$ (aphtitalita) y $\mathrm{K}_{2} \mathrm{SO}_{4} \cdot 2 \mathrm{CaSO}_{4}$ (langbeinita cálcica). Propiciando preferentemente la formación de este último compuesto la atmósfera oxidante del horno.

Obviamente, la presencia de sulfatos alcalinos en el clínker depende, entre otras causas, de la relación de sulfatos y áicalis que aparecen por una u otra razón durante el proceso de clinkerización.

\section{PARTE EXPERIMENTAL (2)}

Partiendo de un clínker portland cuyo análisis y superficie específica se dan en el Cuadro 1, se hicieron diferentes mezclas con yeso dihidrato, yeso hemihidrato y langbeinita cálcica, en las proporciones indicadas en el Cuadro 2.

'Todas las mezclas se homogeneizaron en una mezcladora Túrbula, durante 2 horas.

Las mezclas homogeneizadas se sometieron a tres tipos de conservación: sin meteorización previa; meteorización moderada, y meteorización acelerada.

En el primer caso, se ensayaron las mezclas inmediatamente después de la homogeneización.

En la meteorización moderada, la mezcla homogeneizada se cernió, para que englobara aire húmedo, en una cámara con una h.r. $=88-90 \%$ y a una temperatura de $20 \pm 1^{\circ} \mathrm{C}$; posteriormente se dejaron muy extendidas en bandejas grandes, primeramente durante 2 horas en la misma cámara de humedad donde se cernieron, e inmediatamente después, se conservaron durante 28 horas en una habitación cuya h.r. estaba comprendida entre 65 y $70 \%$, siempre a una temperatura de $20 \pm 1^{\circ} \mathrm{C}$.

Como en el caso anterior, en la meteorización acelerada la mezcla homogeneizada se cernió para que englobará aire húmedo, en las mismas condiciones anteriormente expuestas; inmediatamente después extendidas en grandes bandejas permanecieron durante 24 horas en la misma cámara de humedad (h.r. $=88-90 \%$ y $20 \div 1$ "C).

El ensayo del falso fraguado se realizó de forma similar a la Norma ASTM C451-64T. La relación A/C fue en todo momento 0,32 (3). En el Cuadro 3 se dan los resultados obtenidos.

Paralelamente a estos ensayos se meteorizó, en igualdad de condiciones, langbeinita cálcica obteniendo singenita $\left(\mathrm{K}_{2} \mathrm{SO}_{4} \cdot \mathrm{CaSO}_{4} \cdot \mathrm{H}_{2} \mathrm{O}\right)$ y yeso hemihidrato $\left(\mathrm{CaSO}_{4} \cdot 1 / 2 \mathrm{H}_{2} \mathrm{O}\right)$.

De todas las mezclas ensayadas se tomaron muestras de mezcla anhidra tratada, se tomó y congeló pasta ensayada inmediatamente después de la segunda penetración de la sonda (la congelación se hizo con acetona y alcohol absoluto) y, finalmente, se tomaron muestras de las pastas y se dejaron hidratar durante 24 horas para realizar en todas ellas

(2) Este trabajo se pulicará con mayor amplitud y con nuevos datos, una vez que finalicen tcilos lcs ensayos que se están realizando en la actualidad.

(3) Al fijar A/C no nos hemos atenido a la consistencia fijada por la Norma ASTM C 451-64T porque, según la mezcla realizada. variaba la consistencia. Para simplificar el trabajo se fijó la relación $\mathrm{A}$ 'C. 
análisis por espectroscopía de IR y, en algunas ocasiones, por difracción de rayos X. Los resultados de estos análisis se expresan en los espectrogramas adjuntos.

La explicación que justifica estos hechos sería como sigue:

- La langbeinita reacciona con la humedad del ambiente transformándose en singenita $\left(\mathrm{K}_{2} \mathrm{SO}_{4} \cdot \mathrm{CaSO}_{4} \cdot \mathrm{H}_{2} \mathrm{O}\right)$ y una molécula de $\mathrm{CaSO}_{4}$, según la siguiente reacción:

$$
\mathrm{K}_{2} \mathrm{SO}_{4} \cdot 2 \mathrm{CaSO}_{4}+\mathrm{H}_{2} \mathrm{O} \rightarrow \mathrm{K}_{2} \mathrm{SO}_{4} \cdot \mathrm{CaSO}_{4} \cdot \mathrm{H}_{2} \mathrm{O}+\mathrm{CaSO}_{4}
$$

$\mathrm{El} \mathrm{CaSO}_{4}$ pasaría de forma inmediata a $\mathrm{CaSO}_{4} \cdot 1 / 2 \mathrm{H}_{2} \mathrm{O}$ porque es muy activo, actuando de "germen" en la transformación del hemihidrato que preexiste en el cemento a yeso dihidrato, es decir:

$$
\mathrm{CaSO}_{4} \cdot 1 / 2 \mathrm{H}_{2} \mathrm{O}+1,5 \mathrm{H}_{2} \mathrm{O} \stackrel{\left(\mathrm{CaSO}_{4} \cdot 1 / 2 \mathrm{H}_{2} \mathrm{O}\right)}{\longrightarrow} \mathrm{CaSO}_{4} \cdot 2 \mathrm{H}_{2} \mathrm{O}
$$

En un cemento reciente, es decir, no meteorizado, la langbeinita lógicamente no puede reaccionar con la humedad ambiente; por consiguiente, no tiene lugar la reacción (I) y no existe falso fraguado.

En un cemento sometido a una meteorización acelerada la reacción (II) no se produce durante el amasado ya que el " $\mathrm{CaSO}_{4}$ activo", procedente de la reacción (I), se ha transformado ya en el propio cemento anhidro dando lugar, en todo caso, a un "aterronamiento". Este aparece en ciertos cementos que se mantuvieron defectuosamente conservados.

Algunos investigadores consideran que la singenita es la responsable del aterronamiento. Como queda demostrado en este trabajo, no es precisamente la singenita la causante del "aterronamiento", y sí el " $\mathrm{CaSO}_{4}$ activo" procedente de la langbeinita. La presencia de singenita es una comprobación de que se ha verificado la reacción (I).

\section{CONCLUSIONES}

Las conclusiones que provisionalmente pueden extraerse del trabajo efectuado son las siguientes:

a) La langbeinita cálcica $\left(\mathrm{K}_{2} \mathrm{SO}_{4} \cdot 2 \mathrm{CaSO}_{4}\right)$ formada durante la clinkerización, se hidrata por efecto del aire húmedo, formando singenita $\left(\mathrm{K}_{2} \mathrm{SO}_{4} \cdot \mathrm{CaSO}_{4} \cdot \mathrm{H}_{2} \mathrm{O}\right)$ y "CaSO ${ }_{4}$ activo".

b) En cementos que tienen $\mathrm{CaSO}_{4} \cdot 1 / 2 \mathrm{H}_{2} \mathrm{O}$ como regulador del fraguado (procedente de la deshidratación del $\mathrm{CaSO}_{4} \cdot 2 \mathrm{H}_{2} \mathrm{O}$ en los molinos), aquel " $\mathrm{CaSO}_{4}$ activo" puede actuar como "germen" (en cierto modo, de catalizador) del paso del hemihidrato a yeso $\left(\mathrm{CaSO}_{4} .2 \mathrm{H}_{2} \mathrm{O}\right)$, produciendo falso fraguado.

c) Este falso fraguado puede evitarse utilizando el cemento reciente; es decir, sin meteorizar o, sometiéndole a una aireación prolongada con lo cual el efecto del " $\mathrm{CaSO}_{4}$ activo" se elimina.

d) Una de las causas del "aterronamiento" de un cemento anhidro defectuosamente conservado resulta de la reacción de la langbeinita con la humedad ambiente, al producirse además de singenita, "CaSO ${ }_{4}$ activo" que al reaccionar con los aluminatos del cemento produce, en pequeña proporción, ettringita. Lógicamente hay tamb:én for- 
mación, en muy pequeña cantidad, de fase tobermorítica y portlandita. La singenita no actúa significativamente en este proceso.

e) También se ha comprobado que con la langbeinita en porcentajes de 0,5\% en peso, y menores, en relación al clínker no se producía falso fraguado.

f) Sería necesario estudiar el efecto del aire húmedo insuflado a presión en los propios silos de cemento, como método práctico para evitar el falso fraguado en los cementos que tuvieran las características del estudiado. Para ello sería necesario estudiar el tiempo de tratamiento con el aire húmedo, temperaturas, etc., para evitar la degradación o pérdida de algunas de las propiedades del cemento; principalmente las que pudieran afectar a las resistencias mecán:cas en morteros y hormigones, así como principios y finales de fraguado, éćc.

\section{OBSERVACIONES A LOS ESPECTROS}

\section{Principales zonas diferenciadas:}

Causadas por vibraciones de valencia $\mathrm{OH}=3.000-3.700 \mathrm{~cm}^{-1}$.

En $3.640 \mathrm{~cm}^{-1}$, banda aguda de $\mathrm{OH}^{-}$de portlandita, $\mathrm{Ca}(\mathrm{OH})_{2}$.

Causadas por vibraciones de deformación $\mathrm{H}-\mathrm{O}-\mathrm{H}=1.600-1.700 \mathrm{~cm}^{-1}$.

Causadas por vibraciones $\nu_{3}-\mathrm{CO}_{3}=1.400-1.500 \mathrm{~cm}^{-1}$.

Causadas por $\nu_{3}-\mathrm{SO}_{4}=1.090-1.220 \mathrm{~cm}^{-1}$.

Causadas por $\nu_{3}-\mathrm{SiO}_{4}$ (principalmente $\mathrm{C}_{3} \mathrm{~S}$ y $\mathrm{C}_{2} \mathrm{~S}$ ) $=850-920 \mathrm{~cm}^{-1}$.

La h:dratación del $\mathrm{C}_{3} \mathrm{~S}$ para formar tobermorita se refleja en esta zona por el desplazamiento de la banda hacia más altas frecuencias.

Causadas por $\nu_{4}{ }_{-} \mathrm{SO}_{4}=590-670 \mathrm{~cm}^{-1}$.

En la redacción final del presente trabajo, se desarrollará la interpretación de los espectros IR. Para una mayor información ver, p. ej. la referencia (1).

(1) T. Vázquez; Tesis Doctoral - Universidad Complutense de Madrid: Facultad de Ciencias Químicas. 
CUA D R O 1

Análisis químico

P. F.

R.

$\mathrm{SiO}_{2}$

$\mathrm{Al}_{2} \mathrm{O}_{3} \ldots \ldots \ldots . . .4,6$

$\mathrm{Fe}_{2} \mathrm{O}_{3} \ldots \ldots \ldots . . .3,7$

$\mathrm{CaO} \ldots \ldots \ldots .65,6$

$\mathrm{MgO} \ldots \ldots . .2,5$

$\mathrm{SO}_{3} \ldots \ldots \ldots \ldots \quad 0,6$

$\mathrm{Na}_{2} \mathrm{O} \ldots \ldots \ldots . \quad 0,6$

$\mathrm{K}_{2} \mathrm{O} \ldots \ldots \ldots \ldots, 0,3$

100,00
CUADRO 2

Mezclas estudiadas

\begin{tabular}{|c|c|c|c|}
\hline $\begin{array}{l}\text { Langbeinita } \\
\%\end{array}$ & $\mid \begin{array}{c}\text { Hemihidrato } \\
\%\end{array}$ & Meteorización & $\begin{array}{c}\text { Número de } \\
\text { mezclas }\end{array}$ \\
\hline 0,5 & 4 & $\begin{array}{l}\text { Sin meteorizar ................ } \\
\text { Meteorización moderada } \\
\text { Meteorización acelerada.. }\end{array}$ & $\begin{array}{l}1 \\
1 \\
1\end{array}$ \\
\hline 0,75 & 4 & $\begin{array}{l}\text { Sin meteorizar ............... } \\
\text { Meteorización moderada } \\
\text { Meteorización acelerada }\end{array}$ & $\begin{array}{l}1 \\
1 \\
1\end{array}$ \\
\hline 1,00 & 4 & $\begin{array}{l}\text { Sin meteorizar } \ldots \ldots \ldots \ldots \ldots \\
\text { Meteorización moderada. } \\
\text { Mateorización acelerada }\end{array}$ & $\begin{array}{l}1 \\
1 \\
1\end{array}$ \\
\hline 1,5 & 4 & $\begin{array}{l}\text { Sin meteorizar } \\
\text { Meteorización moderada. } \\
\text { Meteorización acelerada. }\end{array}$ & $\begin{array}{l}1 \\
1 \\
1\end{array}$ \\
\hline 1,75 & 4 & $\begin{array}{l}\text { Sin meteorizar } \\
\text { Meteorización moderada. } \\
\text { Meteorización acelerada }\end{array}$ & $\begin{array}{l}1 \\
1 \\
1\end{array}$ \\
\hline
\end{tabular}

CUAdRO 3

Resultados cualitativos sobre mezclas conteniendo $4 \%$ de hemihidrato

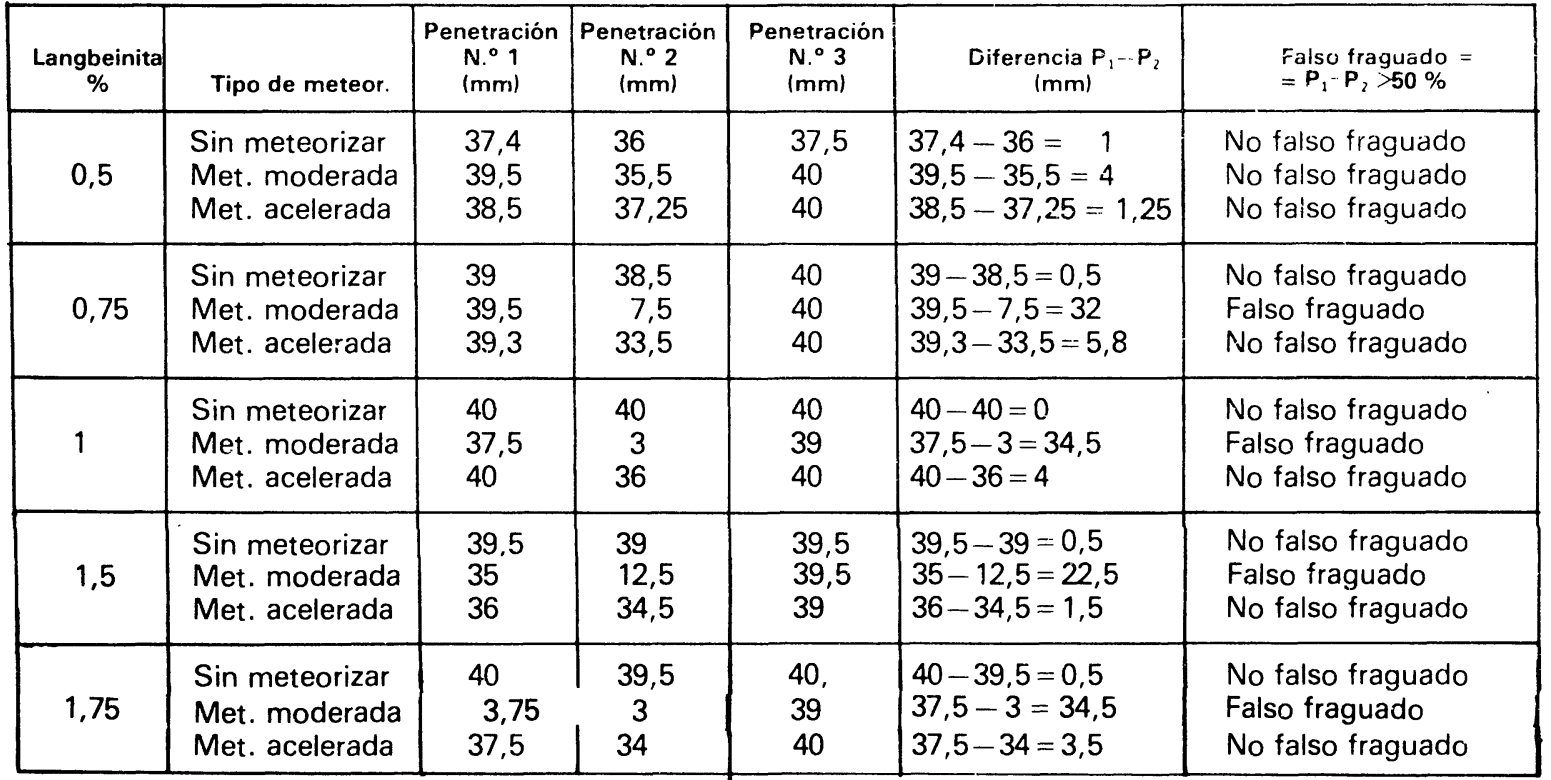

Anexos a los Cuadros 2 y 3

\begin{tabular}{|c|c|c|c|}
\hline $\begin{array}{c}\text { Langbeinita } \\
\%\end{array}$ & $\begin{array}{c}\text { Yeso } \\
\%\end{array}$ & Meteorización & $\begin{array}{c}\text { Numero de } \\
\text { mezclas }\end{array}$ \\
\hline 1 & 4 & Meteorización moderada & 1 \\
\hline
\end{tabular}

\begin{tabular}{|c|c|c|c|c|}
\hline $\begin{array}{c}\text { Penetración } \\
\text { N. } 0^{\circ} 1\end{array}$ & $\begin{array}{c}\text { Penetración } \\
\text { N. }{ }^{\circ} 2\end{array}$ & $\begin{array}{c}\text { Penetración } \\
\mathbf{N} \cdot{ }^{\circ} 3\end{array}$ & $\underset{(\mathrm{mm})}{\text { Diferencia }} P_{1}-P_{2}$ & $\begin{array}{l}\text { Falso fraguado }= \\
P_{1}-P_{2}-50 \%\end{array}$ \\
\hline $40 \mathrm{~mm}$ & $39,5 \mathrm{~mm}$ & $40 \mathrm{~mm}$ & $40-39,5=0,5$ & No falso fraguado \\
\hline
\end{tabular}




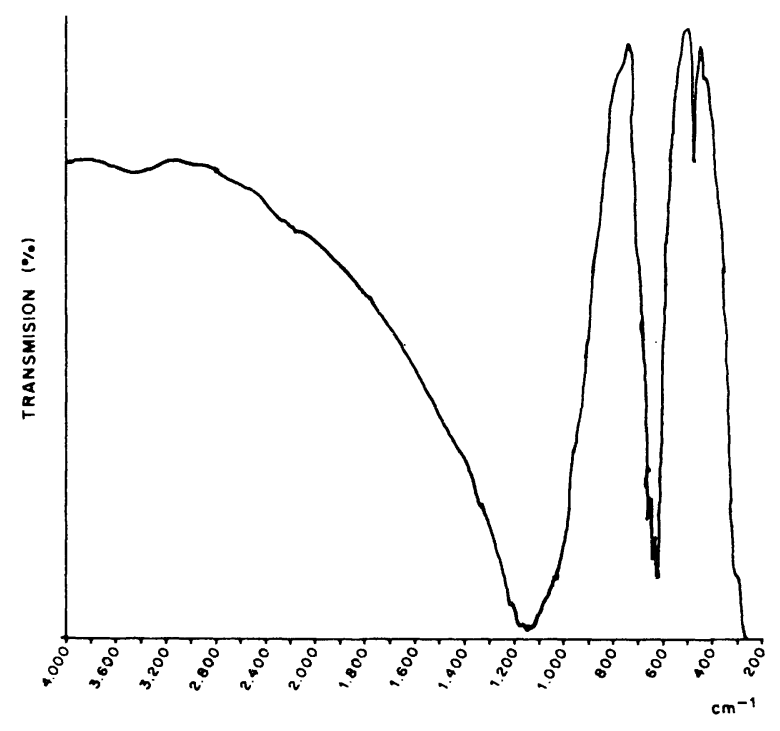

Espectro IR de $\mathrm{K}_{2} \mathrm{SO}_{4} \cdot 2 \mathrm{CaSO}_{4}$.

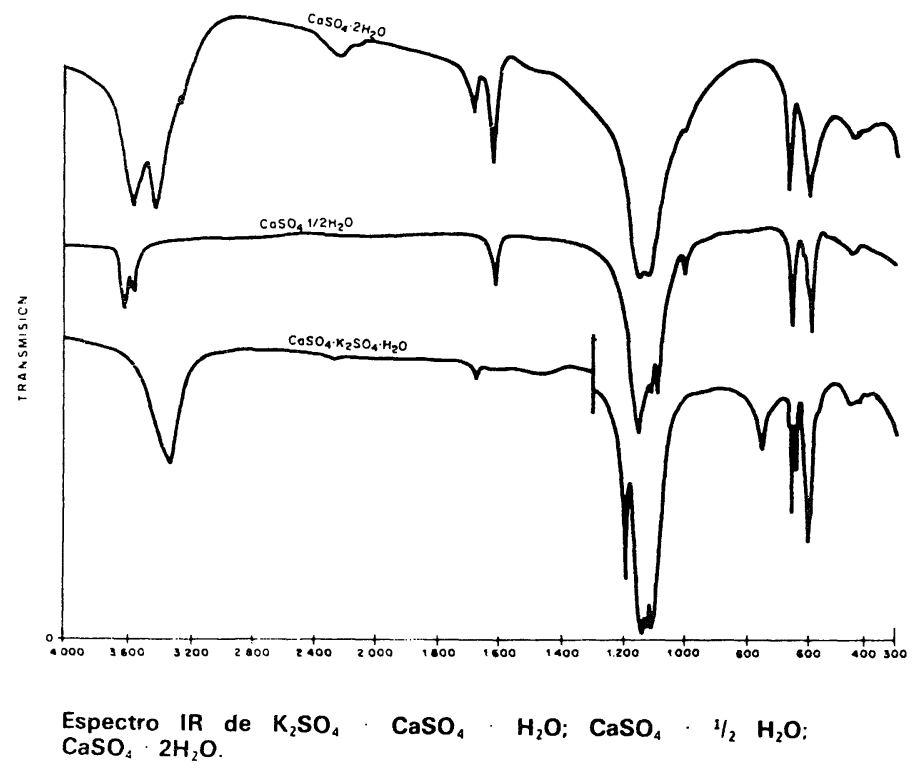

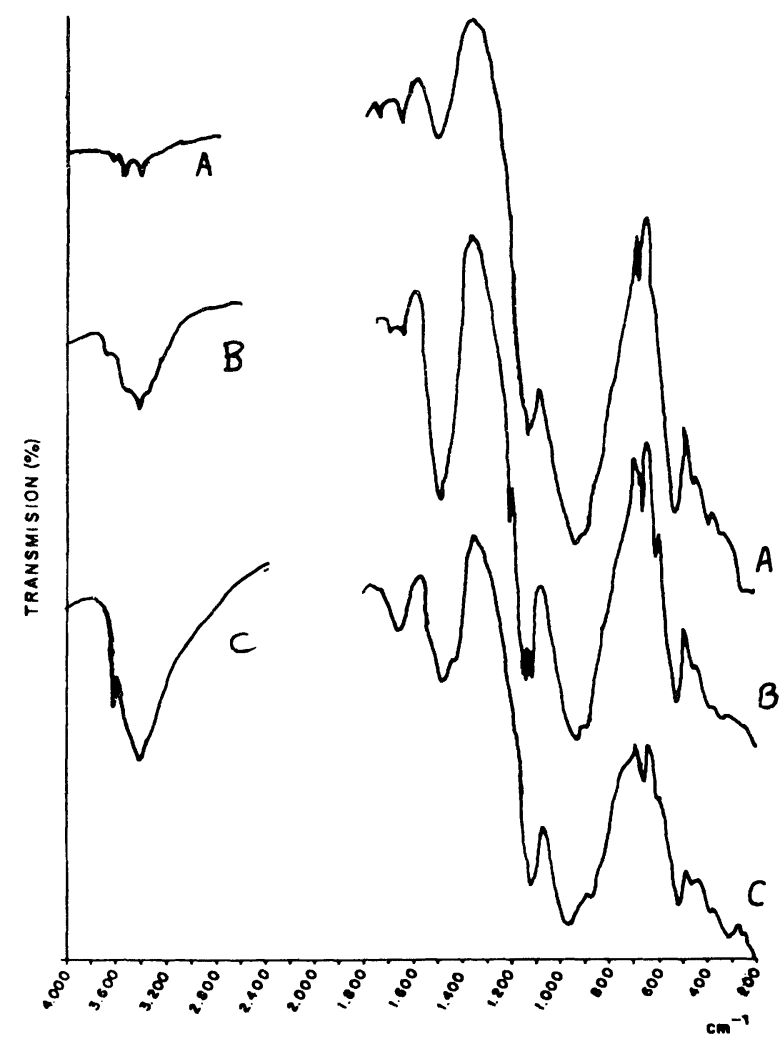

Espectros IR de clinker + $\mathrm{CaSO}_{4} \cdot 1 / 2 \mathrm{H}_{2} \mathrm{O}(4 \%)+\mathrm{K}_{2} \mathrm{SO}$ 2 $2 \mathrm{CaSO}_{4}(1 \%)$

$A=$ Anhidro (sin meteorizar):

$B=$ pasta "congelada») (sin meteorizar)

C = pasta "hidratada» (sin meteorizar).

28

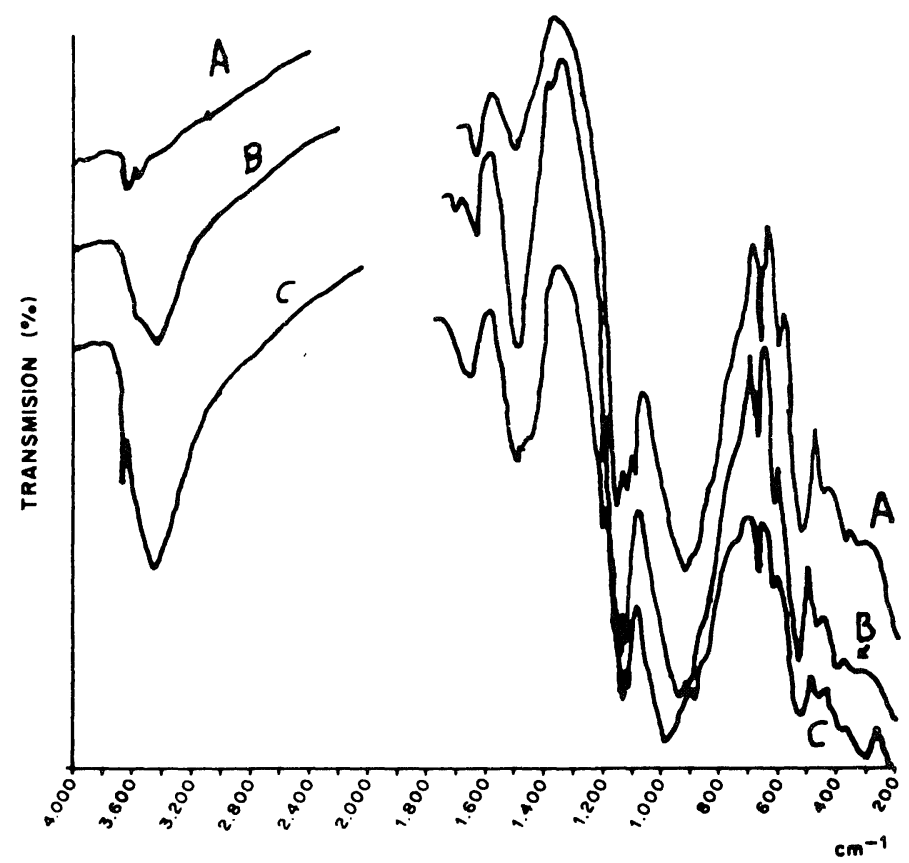

Espectros de IR de clinker + $\mathrm{CaSO}_{4} \cdot 1 / 2 \mathrm{H}_{2} \mathrm{O}(4 \%)+\mathrm{K}_{2} \mathrm{SO}_{4}$ - $2 \mathrm{CaSO}_{4}(1 \%)$

$A=$ Anhidro (meteorización moderada)

B = pasta "congelada" (meteriorización moderada)

$\mathrm{C}=$ pasta uhidratada" (meteriorización moderada)

Materiales de Construccion N." $182-1981$ 


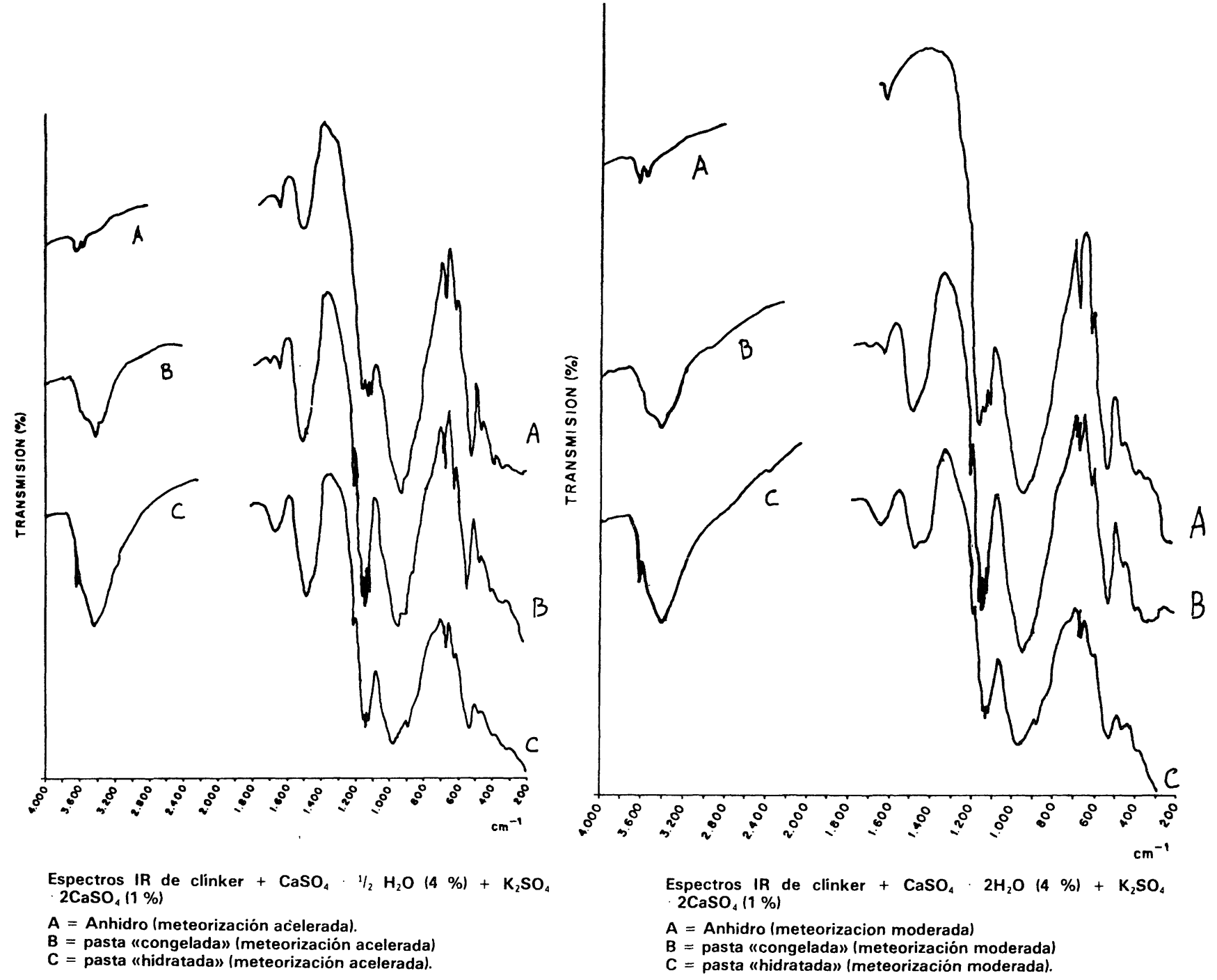

\section{B I B L I O G R A F I A}

W. C. Hansen: "Aireación, la causa del falso fraguado en el cemento Portland"; Material Research and Standards; p. 791; 1961.

J. J. Rowe; G. W. MOREY E I. D. HANSEN : "El sistema binario $\mathrm{K}_{2} \mathrm{SO}_{4}$.CaSO 4 . J. Inor. Chem. V. 27; P. 53$58 ; 1965$.

H. W. Pollit y A. Brown: Proced of the 5. Symposium Chemistry of Cement; V. I.; p. 322 , TOKIO 1968.

H. C. Nrelsen: "Fraguados anómalos del cemento Portland y formación de grumos en el silo"; ZementKaik-Gips; N. 8; p. 380; 1973.

W. Richartz. "Influencia del almacenamiento sobre las propiedades del cemento"; Zement-Kalk-Gips; N.4 2 ; p. $67 ; 1973$.

F. W. Locher: "El fraguado y la resistencia inicial en el cemento", Zement-Kalk-Gips; N.0 2; 1973.

T. VAzQUez MoReno: 40 Aniversario del ITCC; Asamblea General; Desarrollo de Sesiones; p. 33; noviembre de 1974

T. VAzQUez MORENo: "Contribución al estudio de las reacciones de hidratación del cemento Portland, por espectroscopia infrarroja"; Tesis Doctoral; Universidad Complutense de Madrid, Facultad de Ciencias Quimicas; Madrid, 1975.

J. Jawed y J. Skalnz: "Alcalis en el cemento: Una revisión. I. Formas de álcalis sus efectos sobre la formación del clínker"; Cement and Concrete Research; V. 7; p. 719-730; 1977.

Y. B. Pliego Cuervo y F. P. Glasser: "El papel desempeñado por los sulfatos de las reacciones de clinkerización; Formación de la fase y fusión en el sistema CaO-Ca $\mathrm{SiO}_{4}-\mathrm{CaSO}_{4}-\mathrm{K}_{2} \mathrm{SO}_{4}$ "; Cement and Concrete Research; V. 7 ; p. 477-482; 1977.

G. Frigone: "El falso fraguado de la pasta del cemento Portland"; Il Cemento N. 3; p. 207; julio-septiembre, 1978.

B. Cotrin: "Cinética de la hidratación del cemento Portland en presencia de yeso y de sulfato potásico" Il Cemento N. 3; p. 177; julio-septiembre, 1978.

W. KURDowski y Z. Welisex: "Formación de singenita durante la molienda del cemento"; 7.0 Congreso Internacional de la Química del Cemento; V. II; p. I-288; PARIS, 1980. 